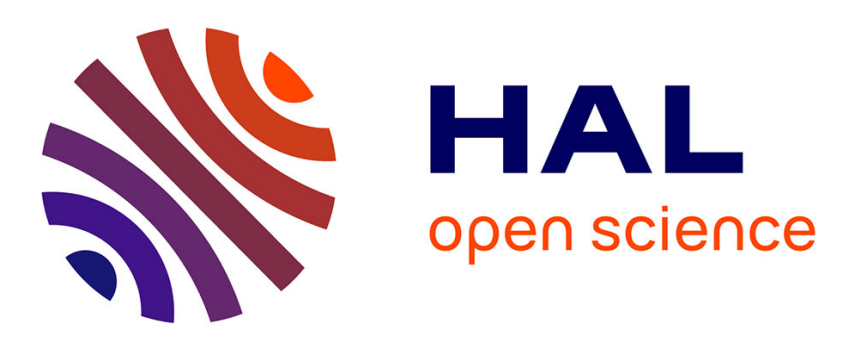

\title{
Low temperature specific heats of permanently densified glassy GeO2
}

Giuseppe Carini, Giovanni Carini, Giovanna d'Angelo, Gaspare Tripodo, Aldo Fontana, Laura Orsingher

\section{- To cite this version:}

Giuseppe Carini, Giovanni Carini, Giovanna d'Angelo, Gaspare Tripodo, Aldo Fontana, et al.. Low temperature specific heats of permanently densified glassy GeO2. Philosophical Magazine, 2010, pp.1. 10.1080/14786435.2010.530617 . hal-00650361

\section{HAL Id: hal-00650361 https://hal.science/hal-00650361}

Submitted on 10 Dec 2011

HAL is a multi-disciplinary open access archive for the deposit and dissemination of scientific research documents, whether they are published or not. The documents may come from teaching and research institutions in France or abroad, or from public or private research centers.
L'archive ouverte pluridisciplinaire HAL, est destinée au dépôt et à la diffusion de documents scientifiques de niveau recherche, publiés ou non, émanant des établissements d'enseignement et de recherche français ou étrangers, des laboratoires publics ou privés. 


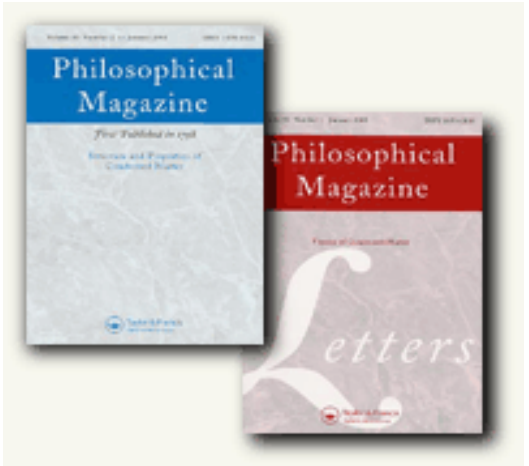

\section{Low temperature specific heats of permanently densified glassy GeO2}

\begin{tabular}{|c|c|}
\hline Journal: & Philosophical Magazine \& Philosophical Magazine Letters \\
\hline Manuscript ID: & TPHM-10-Jun-0285.R1 \\
\hline Journal Selection: & Philosophical Magazine \\
\hline $\begin{array}{r}\text { Date Submitted by the } \\
\text { Author: }\end{array}$ & 03-Sep-2010 \\
\hline Complete List of Authors: & $\begin{array}{l}\text { carini, giuseppe; University of Messina, Dept of Physics } \\
\text { Carini, Giovanni; Dip. Fisica, Università di Messina } \\
\text { D'Angelo, Giovanna; university of messina, physics } \\
\text { Tripodo, Gaspare; Messina University, Physics } \\
\text { Fontana, Aldo; university of trento, physics } \\
\text { Orsingher, Laura; Università di Trento, Fisica }\end{array}$ \\
\hline Keywords: & glass, thermal properties, vibrational properties \\
\hline Keywords (user supplied): & \\
\hline
\end{tabular}

\section{SCHOLARONE Manuscripts}


Measurements of specific heat have been performed over the temperature interval between $0.4 \mathrm{~K}$ and 25 $\mathrm{K}$ in glasses of $\mathrm{GeO}_{2}$, which have been compacted at pressures of $2 \mathrm{GPa}$ and $6 \mathrm{GPa}$ achieving more than $20 \%$ of densification. Above $1 \mathrm{~K}$, the specific heat $C$ is reduced by up to a factor of 2.5 with increasing density, this variation being stronger than that observed below $1 \mathrm{~K}$. The broad peak observed above $1 \mathrm{~K}$ in a $C / T^{3}$ vs. $T$ plot shifts to higher temperatures with increasing density, exhibiting changes which cannot be explained by the modifications of the elastic continuum. In addition to this, the peak shows no changes in shape as a clear indication that the overall distribution of the underlying vibrational modes is independent of density. The temperature dependence of $C$ over the whole explored range is in qualitative agreement with the predictions of the Soft Potential Model.

PACS number: 63.50.+x, 65.40.+g, 78.30.Ly 


\section{Introduction}

Glasses exhibit physical behaviours whose understanding represents a fundamental and complex argument of the condensed matter physics. The nearly linear specific heat $C[1,2]$ observed below a few Kelvin and the subsequent broad hump in $C / \mathrm{T}^{3}$ [3], the Boson peak (BP) which refers to an excess vibrational modes over the classical Debye density of states [3,4] and the plateau of the thermal conductivity $\kappa(T)$ at around $10 \mathrm{~K}$ [5] exhibit a remarkable universality which appears to be quite insensitive to the chemical composition of glasses. It is believed that all these experimental findings have excess low-energy excitations (LEE), which exist beside phonons, as a common origin: LEE, whose investigation is a long-standing problem of considerable interest, take their origin from the disordered topology of amorphous structures. Concerning the low-temperature anomalies observed below $1 \mathrm{~K}$, the successful tunnelling model $[6,7]$ associates the defect modes to single atoms or groups of atoms subjected to quantum-mechanical tunnelling between two different stable positions available in the glassy network and schematizes the locally mobile "particles" by asymmetric double-well potentials. Less clear is the physical origin of the $\mathrm{THz}$ frequency dynamics in glasses causing the $\mathrm{BP}$, the hump in $C / \mathrm{T}^{3}$ and the anomalous characteristics of the phonon transport above few Kelvin. The BP and the broad hump in $C / \mathrm{T}^{3}$ are considered as a manifestation of the enhanced low-energy vibrational dynamics having as a common origin an excess density of vibrational states (DVS), whose nature is a topic, at present very debated $[8,9]$.

A possibility to investigate this matter is to modify the glassy matrix in a controlled way and to observe the correlated changes in different physical properties. This can be obtained by producing structural changes of the glassy network with compaction of the material by high pressures (GPa range). Irreversible compaction of a glass produces significant structural modifications giving rise to a substantial modification of the intermediate range order by cold densification [10]. Permanent densification makes it possible to study indirectly the effects of pressure on physical properties, such as the thermodynamic measurements, for which it is usually difficult to carry out in situ high pressure experiments. Remarkable changes are in fact observed in the linear specific heat [11], the hump in $C / \mathrm{T}^{3}$ and the frequency of the Boson Peak [12-15], and also in the internal friction [16] and the sound velocity [17]. Measurements on permanently densified samples can provide information on the microscopic mechanisms governing the LEE, mainly when the observations are compared with the microscopic structural changes. Permanent densification assumes an important role in the investigation of LEE, also because it permits to get controlled variations of the topology of the glassy network without changing the stoichiometry. Increasing density of $\mathrm{GeO}_{2}$ glasses obtained by cold densification leads to a growing packing of tetrahedral structural units building up the network: the system acquires a structure having a more efficient packing to fill in the lesser volume available to it [18]. These structural changes cause a significant reduction of the local atomic mobility and, consequently, of the anomalies characterizing the 
vibrational and relaxation dynamics $[13,17]$. This could represent an important further step toward a phenomenological understanding of an open question: the possible correlation between the low-energy excitations determining the properties governed by tunnelling systems and those regulating the intermediate-temperature vibrational behaviours, that is the hump in $C / \mathrm{T}^{3}$ and the BP. With the aim of investigating the relation between quantum tunnelling and additional vibrations governing the $\mathrm{THz}$ region, we present here a study concerning the low temperature specific heat in $\mathrm{GeO}_{2}$ glasses, permanently densified by increasing pressures. Differently from the observations in compacted v-SiO where coherent changes of the specific heat were revealed over the whole temperature interval explored [11], the present results show that, above $1 \mathrm{~K}$, the specific heat $C$ is significantly reduced with increasing density exhibiting variations stronger than those observed below $1 \mathrm{~K}$. As explained in the following, these differences are believed to be a consequence of a wider range of investigated densifications (more than $20 \%$ ) which cause more substantial modifications of the intermediate range order.

\section{Experimental Details}

Vitreous germania $\mathrm{GeO}_{2}$ was prepared by the conventional melt-quenching method. Some cuts of the same sample were successfully densified by a multi-anvil HT\&HP (high temperature and high pressure) apparatus. They were compressed at 2 and $6 \mathrm{GPa}$ and heated under pressure at $673 \mathrm{~K}$ for 2 minutes; the pressure was released after a quick cooling down. The final products were homogeneous and permanently densified ingots having both diameter and length of about $5 \mathrm{~mm}$. The densities were measured at room temperature by a Micrometrics Accupyc 1300 gas pycnometer under helium gas with an accuracy of $0.03 \%$, obtaining the values of $3.99 \mathrm{~g} / \mathrm{cm}^{3}(2 \mathrm{GPa})$ and $4.52 \mathrm{~g} / \mathrm{cm}^{3}$ (6 GPa), which correspond to $9 \%$ and $23 \%$ of densification with respect to the density of normal glass $\left(3.66 \mathrm{~g} / \mathrm{cm}^{3}\right)$. After the synthesis and also one year later, the densified $\mathrm{GeO}_{2}$ glasses were characterized by X-ray diffraction which did not reveal any sign of local or extended crystallization. In the following, we will refer to the samples using the pressure at which they were densified.

Longitudinal and shear sound waves were obtained by tuning $\mathrm{X}$ - and Y-cut quartz crystals at their fundamental frequency. The sample-transducer bonding agent was $\mathrm{N}$-Apiezon grease. The sound velocity measurements of longitudinal $\left(\mathrm{v}_{\mathrm{l}}\right)$ and shear $\left(\mathrm{v}_{\mathrm{t}}\right)$ waves were performed at $10 \mathrm{MHz}$ and at room temperature by a pulse-echo technique using an apparatus described elsewhere [19]. The velocity of longitudinal ultrasounds was also measured in all the glasses down to $1.5 \mathrm{~K}$ by using a standard liquid helium cryostat in the range between 1.5 and $20 \mathrm{~K}$ and a cryo-generator above $10 \mathrm{~K}$. Correction which takes into account the bonding was not carried out, but a rough evaluation of the corresponding error introduced in the velocity gave a value less than $0.05 \%$. The low temperature values of shear sound velocities have been evaluated by assuming as temperature independent the value of the ratio $\mathrm{v}_{\mathrm{l}} / \mathrm{v}_{\mathrm{t}}$ obtained at room temperature. 
The specific heat of $\mathrm{GeO}_{2}$ glasses was measured using an automated calorimeter, which operated by the thermal relaxation method, in a ${ }^{3} \mathrm{He}$ cryostat over the range between 0.4 and $2 \mathrm{~K}$ and in a ${ }^{4} \mathrm{He}$ cryostat between 1.5 and $25 \mathrm{~K}$. Samples of about $20-30 \mathrm{mg}$ were bonded to a silicon chip as sample holder by vacuum grease Apiezon $\mathrm{N}$ to optimise the thermal contact. A SMD resistor, as temperature sensor, and a constantan strain gauge, as heater element, were attached to the other chip surface using a thermosetting resin. The good agreement between the measurements taken by the two different experimental setups operating below and above $2 \mathrm{~K}$ is apparent from the figures. The random error and any systematic errors are believed to be less than $3-4 \%$.

\section{Results and discussion}

The experimental data of the specific heat $C(\mathrm{~T})$ obtained between $0.4 \mathrm{~K}$ and $23 \mathrm{~K}$ for normal and densified (at $6 \mathrm{GPa}$ ) $\mathrm{GeO}_{2}$ glasses are compared in Figure 1. The data of normal glass above $3 \mathrm{~K}$ are in very good agreement with earlier experimental observations on vitreous $\mathrm{GeO}_{2}$ [20], reported as a solid line in the same figure. In the overall temperature interval explored, both the samples exhibit an excess $C(\mathrm{~T})$ over the Debye contribution $C_{D}(\mathrm{~T})$, whose behaviour for the $6 \mathrm{GPa}$ glass is reported as a dotted line in Figure 1. When plotted as $C(\mathrm{~T}) / \mathrm{T}^{3}$ vs $\mathrm{T}$ (Figure 2), the specific heat for all the studied samples evidences the characteristic shape for a glass determined by an upturn below $2 \mathrm{~K}$ and a broad peak above $2 \mathrm{~K}$. Growing densification depresses $C(\mathrm{~T})$ over the whole temperature interval explored giving rise to a significant decrease of the hump and to an increase of the peak temperature $\mathrm{T}_{\text {peak }}$, which shifts from about $8.5 \mathrm{~K}$ in normal $\mathrm{GeO}_{2}$, through $11.3 \mathrm{~K}$ in $2 \mathrm{GPa}$ glass, to about $12.6 \mathrm{~K}$ in $6 \mathrm{GPa}$ glass. The Debye contributions (dashed-dotted lines in Figure 2) have been evaluated using the classical relation

$$
C_{D}=\frac{2 \pi^{2} \mathrm{k}_{\mathrm{B}}^{4}}{5 \hbar^{3} \rho}\left[\frac{1}{3}\left(\frac{1}{\mathrm{v}_{l}^{3}}+\frac{2}{\mathrm{v}_{t}^{3}}\right)\right] T^{3}=c_{D} T^{3}
$$

where the term in square bracket is the inverse of the cubic power of the average Debye sound velocity $\mathrm{v}_{\mathrm{D}}$ and the other parameters have their usual meaning. The values of $\mathrm{v}_{\mathrm{D}}$ and some other useful parameters are included in Table 1. In order to compare the changes of the excess specific heat to the modifications of the elastic continuum, we report in a plot $C(\mathrm{~T})$ scaled by the Debye contributions $C_{D}$ of normal and densified $\mathrm{GeO}_{2}$ glasses vs $\mathrm{T} / \Theta_{\mathrm{D}}$ (Figure 3a). The elastic Debye temperatures are: $\Theta_{\mathrm{D}}=305 \mathrm{~K}$ for normal glass, $\Theta_{\mathrm{D}}=336 \mathrm{~K}$ for $2 \mathrm{GPa}$ glass and $\Theta_{\mathrm{D}}=381 \mathrm{~K}$ for $6 \mathrm{GPa}$ glass. It results that the ratio $C(\mathrm{~T}) / C_{D}$ decreases with increasing densification, also exhibiting a well defined shift of the maxima which implies a variation of $T_{\text {peak }}$ stronger than that experienced by $\Theta_{D}$. This finding evidences that $\mathrm{GeO}_{2}$ glass, densified up to more than $20 \%$, shows changes of low-energy vibrational dynamics which are not accounted for by the transformations of the elastic continuum. A further remarkable result is 
given by the comparison of the humps reported in Figure 2 scaled by $\left(\frac{C}{T^{3}}\right)_{\text {peak }}$, see Figure 3 b: despite the significant variations observed in the amplitude and the position of the hump with increasing density, all the scaled curves overlap showing an identical shape. The invariance of the shape leads to conclude that all the vibrational modes, extended and quasi-localized, contributing to the hump form a distribution which is independent of density. Both the observations discussed above are in agreement with the results of studies concerning the low temperature specific heat in compacted $\mathrm{SiO}_{2}$ [11] and in situ high pressure inelastic neutron scattering measurements on poly-isobutylene (PIB) [21,22]. Even if, as pointed out in $\mathrm{SiO}_{2}$ [23], measurements with in situ applied pressure cannot be simply compared to the observations in permanently densified samples retrieved from the same interval of pressure, these results concerning the low energy vibrational dynamics appear to be quite general and independent of the specific experimental conditions.

Among several theoretical models, proposed so far to account for the excess density of vibrational states, one of the most attractive is the Soft Potential Model or SPM [24,25] because it provides an unified description of all the anomalies observed at low (below $1 \mathrm{~K}$ ) and high temperatures. Furthermore, a not negligible detail, it permits a quantitative comparison with the experimental behaviours. In the SPM, the low-frequency vibrational dynamics in a glass is described in terms of localized excitations which coexist with ordinary phonons. These excitations are realized in anharmonic or soft atomic potentials, represented by a fourth degree polynomial:

$$
\mathrm{V}(\mathrm{x})=\mathrm{E}_{0}\left[\eta\left(\frac{\mathrm{x}}{\mathrm{a}}\right)^{2}+\xi\left(\frac{\mathrm{x}}{\mathrm{a}}\right)^{3}+\left(\frac{\mathrm{x}}{\mathrm{a}}\right)^{4}\right]
$$

where $\mathrm{x}$ is an appropriate configurational coordinate having the dimension of a length, $E_{0}$ is of the order of the atomic binding energy, $\eta$ and $\xi$ are small dimensionless parameters with a random distribution of their values. The expression of $V(x)$ includes both double- and single-well potentials. The lowest-energy excitations (double wells) correspond to the well known two level systems (TLS) with a nearly constant density of states. At higher energies there are the quasi-local harmonic oscillators (HO) which occur in single-well potentials and have a density of states following a $\omega^{4}$-dependence. Both the excitations interact with phonons in the same way, the interactions being either resonant or relaxational. At temperatures $\left(\mathrm{T}<<\mathrm{W} / \mathrm{k}_{\mathrm{B}}\right)$ small compared with the energy $\mathrm{W}$, which sets the crossover between TLS and HO excitations, a linear T-dependence for the specific heat is determined by the contribution of TLS's:

$$
\mathrm{C}_{\mathrm{TLS}}(\mathrm{T})=\pi^{2}\left(\frac{2}{9}\right)^{1 / 3} \frac{\mathrm{P}_{0} \eta_{\mathrm{L}}^{5 / 2}}{\mathrm{~W}} \mathrm{k}_{\mathrm{B}}^{2} \operatorname{Tln}^{1 / 3}\left[\frac{\mathrm{W}}{\mathrm{k}_{\mathrm{B}} \mathrm{T}} \sqrt{\frac{\mathrm{t}_{\text {exp }}}{\tau_{\text {min }}(\mathrm{T})}}\right]
$$


where $\mathrm{P}_{0}$ determines the TLS density of states and the other symbols are defined in Ref. [25]. At higher temperatures, a large contribution to the specific heat is provided by HO's whose rapidly increasing density of states leads to a $\mathrm{T}^{5}$ dependence [25]:

$$
\mathrm{C}_{\mathrm{HO}}(\mathrm{T})=\frac{8 \pi^{6} \mathrm{k}_{\mathrm{B}}}{63 \sqrt{2}} \mathrm{P}_{0} \eta_{\mathrm{L}}^{5 / 2}\left(\frac{\mathrm{k}_{\mathrm{B}} \mathrm{T}}{\mathrm{W}}\right)^{5}
$$

The fourth-power HO density of states changes to a density of delocalized vibrational excitations for an energy scale even higher than a characteristic energy $E_{d}$. This is because the average distance between the HO's with $E \geq E_{d}$ becomes smaller than the wavelength of a phonon with about the same energy. Consequently an excitation can directly jump from an oscillator to another producing "delocalization" of HO excitations. The DVS must be reconstructed and a linear dependence on the energy results [25], which corresponds to a $\mathrm{T}^{2}$-dependence for the vibrational specific heat in the high temperature region. Crossover from the low temperature linear dependence to a $\mathrm{T}^{2}$-behaviour, through a $\mathrm{T}^{5}$-dependence, leads to a minimum at a temperature $T_{\min }$ and to a maximum at $\mathrm{T}_{\max } \cong \mathrm{E}_{\mathrm{d}} / 5 \mathrm{k}_{\mathrm{B}}$ in the temperature dependence of $C(\mathrm{~T}) / \mathrm{T}^{3}$. From $\mathrm{T}_{\min }$, the value of the characteristic energy $\mathrm{W}$ can determined: $\mathrm{W} \sim 2$ $\mathrm{k}_{\mathrm{B}} \mathrm{T}_{\min }$.

Following the predictions of SPM, we analyze the specific heat data over the temperature region below $\mathrm{T}_{\max }$ by the relation

$$
\mathrm{C}(\mathrm{T})=c_{1} T+c_{3} T^{3}+c_{5} T^{5}
$$

where the first term in the r. h. s. of Eq. (4) refers to TLS contribution, the second one to the overlap of the elastic Debye contribution and the eventual excess usually observed in glasses [26], and finally the third term evaluates the HO contribution. The fits, reported in Figure 2 as dotted lines, reproduce quite well the experimental curves up to 5-7 $\mathrm{K}$ giving values of the parameters which are included together with those of $\mathrm{T}_{\min }=\left(\frac{c_{1}}{c_{5}}\right)^{\frac{1}{4}}$ in Table 1. The values of $\mathrm{c}_{1}, \mathrm{c}_{3}$ and $\mathrm{c}_{\mathrm{D}}$ derived for normal glass are in good agreement with those previously obtained by Stephens [27]. We like to remark, however, that a more exhaustive analysis of the TLS contribution should require a study extended to lower temperatures than those available in the present experiment and, as a consequence of the restricted temperature range, our considerations are to be considered as highly tentative. Inspection of the behaviour of the parameters of the fits and of the maximum of the peak, $\left(\frac{C}{T^{3}}\right)_{\text {peak }}$, in densified glasses shows that growing densification appears to be more efficient in depressing the LEE contributing to the hump than TLS responsible for nearly linear specific heat below $1 \mathrm{~K}$. In fact, $\left(\frac{C}{T^{3}}\right)_{\text {peak }}$ is reduced by up to a factor of 2.5 in the $6 \mathrm{GPa}$ 
glass, while $\mathrm{c}_{1}$ by a factor of 1.25 . This result is quite different from the observations in compacted vitreous $\mathrm{SiO}_{2}$ [11], where $C$ is reduced by about the same factor over the whole temperature range explored $(0.1 \mathrm{~K}<\mathrm{T}<35 \mathrm{~K})$, and suggests a possible different microscopic origin for the low energy excitations giving rise to TLS and to the hump (or the BP) in $\mathrm{GeO}_{2}$ glass. Previous neutron and $\mathrm{x}$-ray diffraction studies $[18,28]$ of densified $\mathrm{GeO}_{2}$ glasses gave strong evidence for a reduction in the length scale of the intermediate-range order induced by cold densification up to $10 \mathrm{GPa}$, excluding a transition from a $\mathrm{GeO}_{4}$ tetrahedral to a $\mathrm{GeO}_{6}$ octahedral structure. The densification process has been described in terms of a step-like behavior: in the first step there is a decrease in the sizes of the network cages, associated with a closer packing of the relatively rigid tetrahedra [28]. Further increase of pressure leads to a reconfiguration of the system via a rotation of the tetrahedra around the Ge-O-Ge bonds, which causes a distortion of the $\mathrm{GeO}_{4}$ tetrahedra and a more efficient packing of oxygen ions to fit to the growingly reduced volume [18]. These structural changes should hinder the local mobility of $\mathrm{GeO}_{4}$ tetrahedra, causing a significant reduction of low energy excitations (soft vibrations and tunneling states) and also a shift to higher frequencies of the local vibrational modes involved.

An interesting consideration concerns the density variations of the energy crossover W, which is related to the other SPM's parameters by [25]:

$$
\mathrm{W} \cong \frac{\hbar^{\frac{4}{3}} \rho^{\frac{4}{9}} V^{\frac{2}{3}}}{\bar{M}^{\frac{7}{9}}}\left(\frac{\overline{\mathrm{M}}}{\mathrm{M}}\right)^{\frac{2}{3}}
$$

where $\bar{M}$ is the average mass of the atoms constituting the glass (for $\mathrm{GeO}_{2}, \bar{M}=34.87$ a.u.), $\mathrm{M}$ the effective mass of the moving group, $\rho$ the density and $V$ the sound velocity. In order to evaluate the ratio $\frac{M}{\bar{M}}$, we use the values of $\rho, \mathrm{V}$ and $\mathrm{T}_{\min }$ (determining the energy $\mathrm{W}$ ) reported in Table 1. The SPM does not distinguish between longitudinal and shear sound velocity and, tentatively, we use the average Debye sound velocity. The obtained values of $\frac{M}{\bar{M}}$ decreases from 6.5 in normal glass to 4.5 in $6 \mathrm{GPa}$ sample pointing to an apparent reduction of the effective mass of the moving group.

It is concluded that densification at elevated pressures of $\mathrm{GeO}_{2}$ glasses causes network modifications associated to the formation of more packed and tied groups of atoms and depresses the local mobility of the units which are the source of additional low-energy excitations over the contribution of the ordinary elastic waves. The reduced mobility of these "quasi-particles" leads to growingly smaller contributions to the vibrational specific heat and to the scattering of thermal phonons which are responsible for the heat transport in a glass. Strong support for the latter consideration is given by the low temperature behaviour of the thermal conductivity $\kappa$ in a densified $\mathrm{SiO}_{2}$ glass: a substantial increase of the magnitude 
of the plateau characterizing $\kappa$ in normal vitreous silica is observed, corresponding to an increase of the phonon mean free path [29]. By exploring an interval of densifications (up to $23 \%$ ) quite larger than the one investigated in $\mathrm{v}^{-\mathrm{SiO}_{2}}$ (up to about $11 \%$ [11]), it has been possible to disclose the following peculiarities:

(i) the modifications of medium range structure give rise to a more severe reduction of additional soft vibrations involved in the excess specific heat than that experienced by TLS;

(ii) the hardening of the elastic continuum with increasing densification is not sufficient to account for the changes observed in the excess $C$;

(iii) the invariance of the shape of $C / T^{3}$ with increasing densification indicates that the vibrational modes causing the low temperature specific heat cannot be separated in extended and quasilocalized modes, because they are hybridized and form an overall distribution independent of densification.

\section{Conclusions.}

A study of low temperature specific heat $C$ in permanently densified $\mathrm{GeO}_{2}$ glasses has revealed an excess $C$ over that predicted by the Debye theory which decreases with increasing densification. Between $2 \mathrm{~K}$ and $25 \mathrm{~K}, C / \mathrm{T}^{3}$ shows a hump which shifts towards higher temperatures and decreases by up to a factor of 2.5 in the glass compacted at $6 \mathrm{GPa}$. Below $2 \mathrm{~K}$, an additional contribution is also observed: $C$ follows an approximately linear temperature dependence disclosing a well-definite reduction by up to a factor of 1.25 with increasing densification. These observations lead to conclude that glass densification reduces the excess density of low-energy vibrational states over the Debye prediction, affecting also, but to a less extent, the density of two-level systems which are the source for the linear term. Moreover the results prove unambiguously that the density variations of the low-energy vibrational dynamics cannot be accounted for by the modifications of the elastic continuum.

Analysis of experimental behaviours reveals that they are in qualitative agreement with the predictions of the Soft Potential Model. 


\section{References}

[1] Amorphous Solids: Low Temperature Properties, W. A. Phillips, ed., Springer-Verlag, Berlin, 1981.

[2] R. O. Pohl, X. Liu, E. Thompson, Rev. Mod. Phys. 74, 991 (2002) and references therein.

[3] G. D’Angelo, G. Carini, C. Crupi, M. Koza, G. Tripodo, C. Vasi, Phys. Rev. B 79, 014206 (2009).

[4] T. Nakayama, Rep. Prog. Phys. 65, 1195 (2002).

[5] G. D’Angelo, C. Crupi, G. Salvato, G. Tripodo, C. Vasi, J. Phys. Chem. B 114, 2467 (2010).

[6] P. W. Anderson, B. I. Halperin and C. Varma, Phil. Mag. 25, 1 (1972).

[7] W. A. Phillips, J. Low Temp. Phys. 7, 1657 (1972).

[8] H. Shintani and H. Tanaka, Nature Mat. 7, 870 (2008).

[9] G. Monaco and V. M. Giordano, PNAS 106, 3659 (2009).

[10] S. Susman et al, Phys. Rev. B 43, 1194 (1991).

[11] X. Liu, H. v. Lohneysen, G. Weiss, J. Arndt, Z. Phys. B 99, 49 (1995).

[12] Y. Inamura et al, Physica B 263-264, 299 (1999)

[13] L. Orsingher, A. Fontana, G. Carini Jr, G. Carini, G. Tripodo, T. Unruh, U. Buchenau, J. Chem. Physics. 132, 124508 (2010).

[14] O. Pilla, L. Angelani, A. Fontana, R. Goncalves, G. Rocco, J. Phys.: Cond. Matter 15, S995 (2003).

[15] O. Pilla, S. Caponi, A. Fontana et al, J. Phys.: Cond. Matter 16, 8519 (2004).

[16] G. Weiss, A. Dau, M. Sohn, J. Arndt, Physica B 219-220, 290 (1996).

[17] G. Carini Jr., L. Orsingher, G. Tripodo and E. Gilioli, Phil Mag. 88, 4143-4150 (2008).

[18] S. Sampath, C. J. Benmore, K. M. Lantzky, J. Neuefeind, K. Leinenweber, D. L. Price, and J. L. Yarger, Phys. Rev. Lett. 90, 115502 (2003).

[19] G. Carini and F. Mento, J. Phys. E 12, 259 (1979).

[20] A. P. Jeapes, A. J. Leadbetter, C. G. Waterfield, K. E. Wycherley, Phil Mag. 29, 803 (1974).

[21] K. Niss et al., Phys. Rev. Lett. 99, 055502 (2007)

[22] L. Hong et al., Phys. Rev. B 78, 134201 (2008)

[23] R. J. Hemley, C. Meade, and H. K. Mao, Phys. Rev. Lett. 79, 1420 (1997)

[24] V. G. Karpov, M. I. Klinger, F. N. Ignatiev, Zh. Expt. Teor. Fiz. 84, 760 (1983) [Sov. Phys. JEPT 57, 439 (1983)].

[25] D. A. Parshin, Phys. Solid State 36, 991 (1994); Phys. Rev. B49, 9400 (1994).

[26] R. O. Pohl, Low temperature specific heat of glasses, in Amorphous Solids: Low Temperature Properties, W. A. Phillips, ed., Springer-Verlag, Berlin, 1981, p. 27.

[27] R. B. Stephens, Phys. Rev. B13, 853 (1976).

[28] C. E. Stone et al., J. of Non-Cryst. Sol. 293-295, 769 (2001)

[29] Da-Ming Zhu, Phys. Rev. B50, 6053 (1994) 
Table 1. Parameters of normal and densified $\mathrm{GeO}_{2}$ glasses. The values of the density $\rho$ are taken at room temperature. The values of longitudinal $\left(\mathrm{V}_{1}\right)$ and shear $\left(\mathrm{V}_{\mathrm{t}}\right)$ sound velocities at $1.5 \mathrm{~K}$ are included together with the calculated Debye sound velocity $\mathrm{V}_{\mathrm{D}}$.

\begin{tabular}{|c|c|c|c|c|c|c|c|c|c|c|}
\hline $\begin{array}{c}\mathrm{GeO}_{2} \\
\text { glasses }\end{array}$ & $\begin{array}{c}\rho \\
\left(\mathrm{kg} \mathrm{m}^{-3}\right)\end{array}$ & $\begin{array}{c}V_{l} \\
\left(\mathrm{~m} \mathrm{~s}^{-1}\right)\end{array}$ & $\begin{array}{c}\mathrm{V}_{\mathrm{t}} \\
\left(\mathrm{m} \mathrm{s}^{-1}\right)\end{array}$ & $\begin{array}{c}V_{D} \\
\left(\mathrm{~m} \mathrm{~s}^{-1}\right)\end{array}$ & $\begin{array}{c}\mathbf{c}_{\mathrm{D}} \\
\left(\mu \mathrm{J} / \mathbf{g K}^{4}\right)\end{array}$ & $\begin{array}{c}\mathbf{c}_{1} \\
\left(\mu \mathbf{J} / \mathbf{g K}^{2}\right)\end{array}$ & $\begin{array}{c}\mathbf{c}_{3} \\
\left(\mu \mathbf{J} / \mathbf{g K}^{4}\right)\end{array}$ & $\begin{array}{c}\mathbf{c}_{5} \\
\left(\mu \mathrm{J} / \mathbf{g K}^{6}\right)\end{array}$ & $\begin{array}{l}\mathbf{T}_{\text {min }} \\
(\mathbf{K})\end{array}$ & $\begin{array}{l}\left(\frac{C}{T^{3}}\right)_{\text {peak }} \\
\left(\mu \mathbf{J} / \mathbf{g K}^{\mathbf{4}}\right)\end{array}$ \\
\hline normal & 3660 & 3832 & 2328 & 2572 & 1.96 & 0.8 & 2.1 & 0.094 & 1.71 & 5.18 \\
\hline $2 \mathrm{GPa}$ & 3994 & 3992 & 2495 & 2748 & 1.47 & 0.7 & 1.48 & 0.038 & 2.07 & 3.32 \\
\hline $6 \mathrm{GPa}$ & 4520 & 4502 & 2701 & 2988 & 1.01 & 0.64 & 1.1 & 0.015 & 2.55 & 2.12 \\
\hline
\end{tabular}




\section{Figure Captions}

Figure 1. The temperature dependence of the specific heat $C$ in normal $(O)$ and densified at $6 \mathrm{GPa}(\nabla)$ $\mathrm{GeO}_{2}$ glasses. Earlier experimental observations on normal vitreous $\mathrm{GeO}_{2}$ [20] are reported as a solid line for comparison. Dashed-dotted line indicates the elastic Debye contribution $C_{D}$ of densified glass calculated from sound velocity data. Dotted line marks the super-linear contribution observed below 0.8 K.

Figure 2. The temperature dependence of $C(\mathrm{~T}) / \mathrm{T}^{3}$ for densified $\mathrm{GeO}_{2}$ glasses: normal glass, (O); 2 GPaglass, $(\mathbf{\Delta})$; 6 GPa-glass, $(\nabla)$. The dashed-dotted lines show the elastic Debye contributions calculated from sound velocity data. Dotted lines are the fits to Eq. (4).

Figure 3. (a) Comparison of the specific heats of densified $\mathrm{GeO}_{2}$ glasses, plotted as $C / C_{D}$ vs $\mathrm{T} / \Theta_{\mathrm{D}}$ : normal glass, (O); 2 GPa-glass, ( $\mathbf{\Delta}) ; 6$ GPa-glass, $(\nabla)$.

(b) Comparison of the specific heats of densified $\mathrm{GeO}_{2}$ glasses, plotted as $C(\mathrm{~T}) / \mathrm{T}^{3}$ scaled by $\left(\frac{C}{T^{3}}\right)_{\text {peak }} \underline{\text { vs }}$ T/T $\mathrm{T}_{\text {peak }}$ : normal glass, (O); 2 GPa-glass, $(\boldsymbol{\Delta}) ; 6$ GP-glass, $(\nabla)$. 
Fig. 1

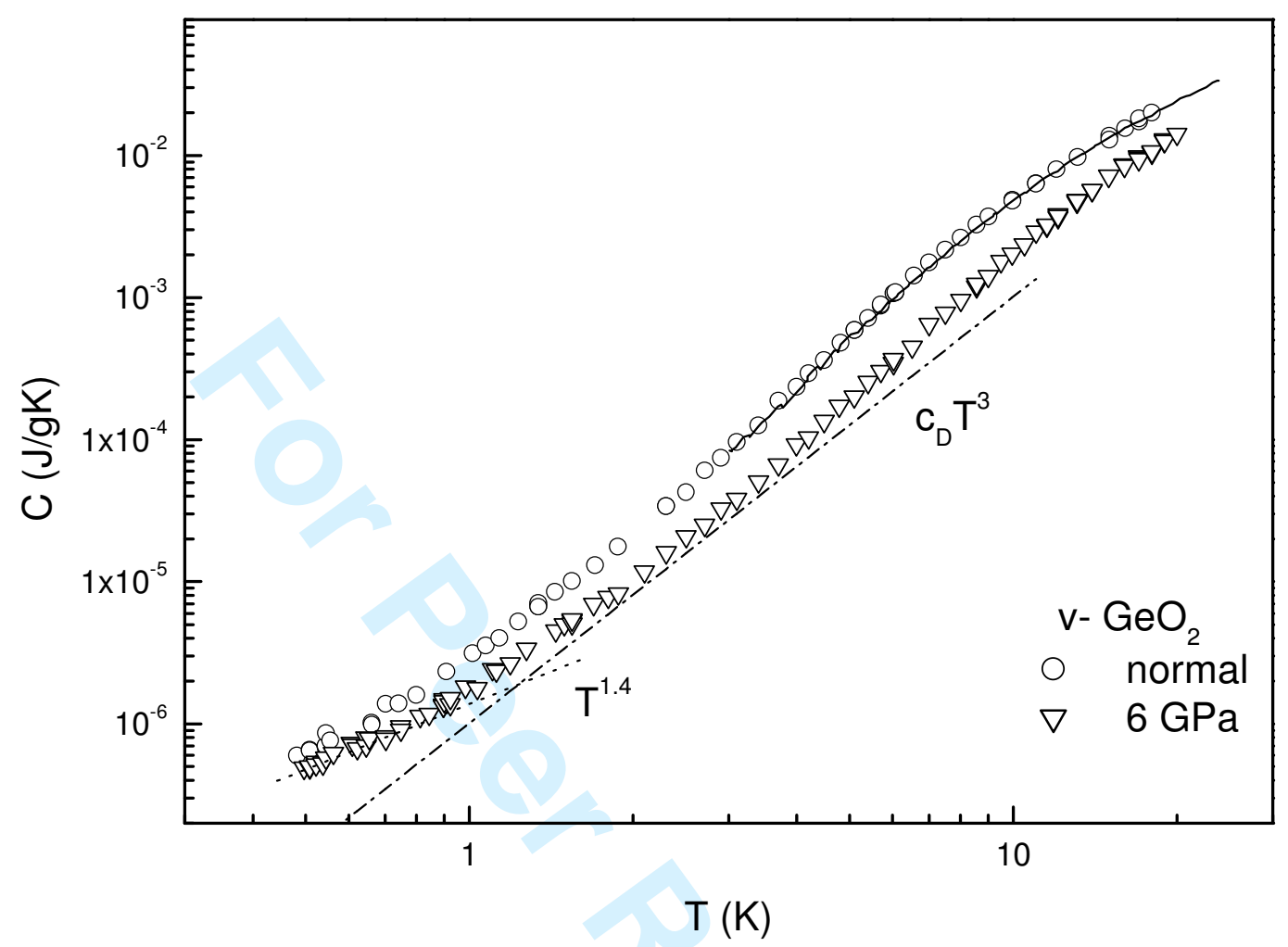


Fig. 2

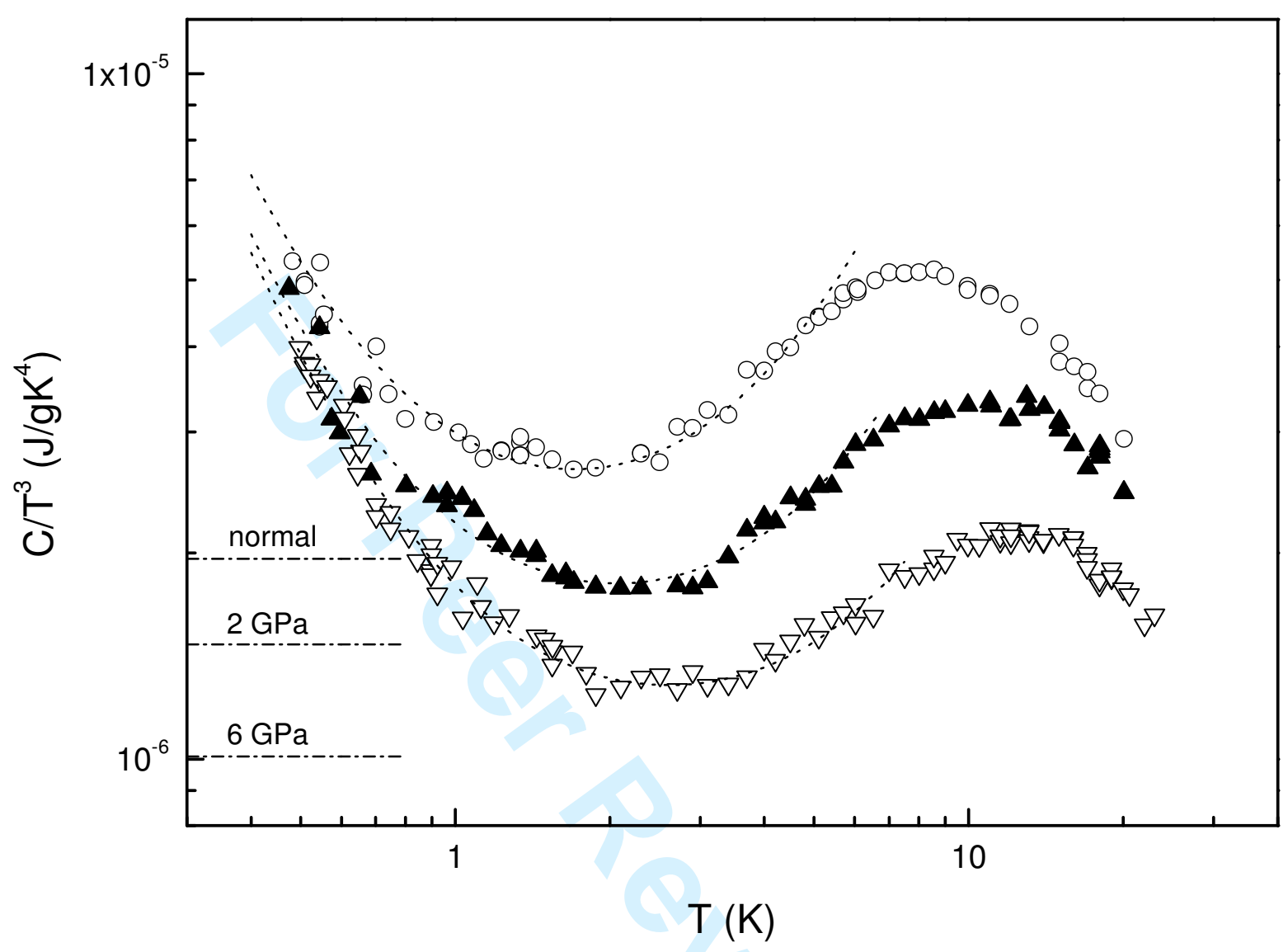


Fig. 3
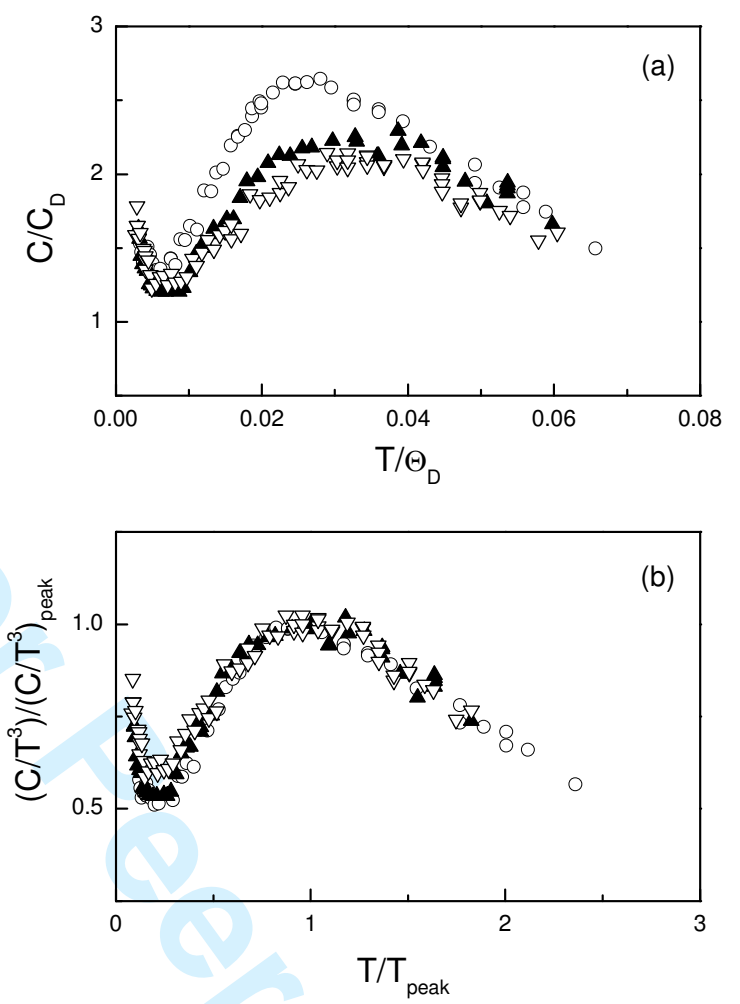